\section{Intensive blood pressure reduction may reduce hematoma growth after ICH}

Some evidence indicates that early lowering of blood pressure (BP) after acute intracerebral hemorrhage $(\mathrm{ICH})$ is beneficial, but data from randomized trials are lacking. Now, in a randomized pilot trial designed as a run-in to a larger study, Anderson et al. demonstrate the feasibility of intensive early lowering of BP after $\mathrm{ICH}$ and show that such treatment seems to reduce hematoma growth.

The researchers randomized 404 patients with acute spontaneous $\mathrm{ICH}$ diagnosed within $6 \mathrm{~h}$ of onset to either early intensive lowering of BP (target systolic BP $140 \mathrm{mmHg}$ ) or standard guideline-based management of BP (target systolic BP $180 \mathrm{mmHg}$ ). Systolic BP was significantly lower in the intensive-intervention group than in the standard-management group at every time point investigated from $30 \mathrm{~min}$ to 7 days after randomization $(P<0.0001$ for all). At $24 \mathrm{~h}$, the mean proportional hematoma growth in the intensive-intervention group was significantly lower than that in the standard-management group ( $P=0.04)$; however, the significance of this difference disappeared following adjustment for initial hematoma volume and time from ICH onset to CT scanning $(P=0.06)$. The mean absolute increase in hematoma volume was smaller in the intensive-intervention group than in the standard-management group, but the difference was not significant. Intensive BP lowering did not influence the risk of adverse events at 90 days.

Following these promising results, the researchers are conducting a larger trial to determine the effects of intensive BP lowering on clinical outcomes in 2,800 patients with $\mathrm{ICH}$.

Original article Anderson CS et al. (2008) Intensive blood pressure reduction in acute cerebral haemorrhage trial (INTERACT): a randomised pilot trial. Lancet Neurol 7: 391-399

\section{Cholinergic drugs might be effective in patients with cerebellar degenerative disorders}

Changes in the ascending cholinergic neurons of the brain might contribute to the symptoms of neurodegenerative disorders. Hirano et al. studied brain acetylcholinesterase (AChE) activity in patients with cerebellar degenerative disorders in order to establish the pattern of cholinergic activity in these conditions.

This Japanese study enrolled 8 patients with the cerebellar variant of multiple system atrophy (MSA-C), 7 patients with spinocerebellar ataxia type 3 (SCA3), 3 patients with spinocerebellar ataxia type 6 (SCA6), and 13 healthy controls. AChE activity in various brain regions was measured with PET and three-dimensional T1-weighted MRI.

Mean thalamic AChE activity was significantly less in patients with MSA-C and in those with SCA3 than in controls $(P<0.01$ for both) and showed an inverse correlation with Unified Parkinson's Disease Rating Scale (UPDRS) motor and axial scores in patients with SCA3 $(P \leq 0.001)$, suggesting that impairment of thalamic cholinergic neurons contributes to motor symptoms in these conditions. In addition, patients with MSA-C showed decreased AChE activity in the posterior lobe of the cerebellar cortex. No significant alterations in AChE activity were seen in the neocortex of patients with MSA-C, SCA3 or SCA6, indicating that in these patients frontal lobe dysfunction does not result from cholinergic deficits.

On the basis of the observed dysfunction in the brain cholinergic system, the authors suggest that cholinergic modulating drugs might improve ataxia and possibly other symptoms in patients with MSA-C or SCA3.

Original article Hirano $S$ et al. (2008) PET study of brain acetylcholinesterase in cerebellar degenerative disorders. Mov Disord [doi:10.1002/mds.22056] 